Review Paper

\title{
Permanent Magnetic Fluids
}

\author{
${ }^{1}$ Nicolae Petrescu and ${ }^{2}$ Florian Ion Tiberiu Petrescu \\ ${ }^{1}$ Bucharest University, Bucharest, (CE), Romania \\ ${ }^{2}$ ARoTMM-IFToMM, Bucharest Polytechnic University, Bucharest, (CE), Romania
}

\section{Article history}

Received: 26-07-2019

Revised: 21-08-2019

Accepted: 22-08-2019

Corresponding Author Florian Ion Tiberiu Petrescu ARoTMM-IFToMM, Bucharest Polytechnic University, Bucharest, (CE), Romania Email: scipub02@gmail.com
Abstract: A team of researchers at the University of Massachusetts Amherst has accidentally obtained the first magnetized permanent liquid in the world, the droplets of this liquid moving in unison and able to associate in different forms while being manipulated exterior by means of a magnet, according to a new study published in the latest issue of the journal Science, reports Agerpres. We usually imagine magnets as being in a solid-state of aggregation, "says Thomas Russell, professor of polymers and engineering at the University of Massachusetts Amherst. But we now know that "we can get liquid magnets that can take different forms - and we can decide the forms they take," he added, specifying that droplets in this magnetic fluid can form spheres, cylinders, or the shape of a flat disc of the pancake dough. "We can make them look like a sea hedge if we want," he said. Russell and his team obtained this liquid magnet by chance while experimenting with a 3D printer. They have tried to print liquids in order to obtain new solid materials, but having the properties of fluids, for various applications in the field of energy. One day, the postdoctoral student and coordinator of this study, Xubo Liu, noticed that droplets of the resulting 3D material, made up of magnetized iron oxide particles, rode unison on magnetic support. Subsequently, his team noticed that the entire construct, not just those particles, had become magnetic. Using a specially customized 3D printer for liquids, the team managed to generate millimeters drops of water, oil and iron oxides. These drops retain their shape because of some of the iron oxide particles in the composition form bonds with the surfactants (also called tensides substances that reduce the surface tension of the liquid). Surfactants form a film around the water and some iron oxide particles enter the composition of the film, while the rest remain inside, as Russell explains. Then the team placed these droplets near a magnetic coil to magnetize them. After removing the magnetic coil, droplets have shown unprecedented behavior to liquid substances so far-they have retained their magnetic properties. Magnetic fluids were known to physicists. They are called ferrofluids and are fluids consisting of ferromagnetic, ferromagnetic or paramagnetic colloidal particles suspended in a carrier liquid. Characteristic of ferrofluids is that they remain magnetized only in the presence of a magnetic field. When the droplets were close to the magnetic field, the iron oxide particles aligned unison, indicating the same direction. Once the magnetic field has been stopped, the iron oxide particles have joined the surfactant in the film in tight formation, without being able to move and thus keep their alignment. The iron oxide particles that have remained floating inside the liquid have also taken this alignment. Scientists still do not understand the mechanism by which these particles remain magnetized. Once they have this explanation, the applications of this discovery can be multiple, both in the field of energy, robotics and space programs.

Keywords: Magnets, Magnetic Fluids, Magnetic Properties, Magnetized Permanent Liquid, Elementary Particle Dynamics, Condensed Matter, Spin Kinetic Energy 


\section{Introduction}

Liquids also have a lasting memory that has been suspected for a long time, especially after studies on water and its different forms of crystallization.

A team of researchers at the University of Massachusetts Amherst has accidentally obtained the first magnetized permanent liquid in the world, the droplets of this liquid moving in unison and able to associate in different forms while being manipulated exterior by means of a magnet, according to a new study published in the latest issue of the journal Science, reports Agerpres.

We usually imagine magnets as being in a solid-state of aggregation, "says Thomas Russell, professor of polymers and engineering at the University of Massachusetts Amherst. But we now know that "we can get liquid magnets that can take different forms - and we can decide the forms they take," he added, specifying that droplets in this magnetic fluid can form spheres, cylinders, or the shape of a flat disc of the pancake dough. "We can make them look like a sea hedge if we want," he said.

Russell and his team obtained this liquid magnet by chance while experimenting with a 3D printer. They have tried to print liquids in order to obtain new solid materials, but having the properties of fluids, for various applications in the field of energy.

One day, the postdoctoral student and coordinator of this study, Xubo Liu, noticed that droplets of the resulting 3D material, made up of magnetized iron oxide particles, rode unison on magnetic support. Subsequently, his team noticed that the entire construct, not just those particles, had become magnetic.

\section{How the Liquid Magnet Works}

Using a specially customized 3D printer for liquids, the team managed to generate millimeters drops of water, oil and iron oxides. These drops retain their shape because of some of the iron oxide particles in the composition form bonds with the surfactants (also called tensides-substances that reduce the surface tension of the liquid). Surfactants form a film around the water and some iron oxide particles enter the composition of the film, while the rest remain inside, as Russell explains.

Then the team placed these droplets near a magnetic coil to magnetize them. After removing the magnetic coil, droplets have shown unprecedented behavior to liquid substances so far-they have retained their magnetic properties.

Magnetic fluids were known to physicists. They are called ferrofluids and are fluids consisting of ferromagnetic, ferromagnetic or paramagnetic colloidal particles suspended in a carrier liquid. Characteristic of ferrofluids is that they remain magnetized only in the presence of a magnetic field.

When the droplets were close to the magnetic field, the iron oxide particles aligned unison, indicating the same direction. Once the magnetic field has been stopped, the iron oxide particles have joined the surfactant in the film in tight formation, without being able to move and thus keep their alignment. The iron oxide particles that have remained floating inside the liquid have also taken this alignment.

Scientists still do not understand the mechanism by which these particles remain magnetized. Once they have this explanation, the applications of this discovery can be multiple, both in the field of energy, robotics and space programs (Berkeley, 2019).

\section{Issues}

Behind the apparent simplicity of water, reflected in the most famous of chemical formulas, there is an amazingly sophisticated molecule, responsible for perhaps the most exhausting "battle" in the history of science. More than 150 years after the "hostilities" began, the controversy over water memories came back to date.

Has water memory? Can a diluted liquid 120 times preserve the properties of the initial solution? If the incredible becomes credible then it means that water can somehow "remember" the qualities of the dissolved compounds in it even when they have not even left a molecule. And demonstrating that water has memory is to confirm the principles of homeopathy, because ultra diluted remedies are one of the decisive elements of this therapy, relying precisely on the ability of water to retain in its structural matrix the energy footprint or the vibrational signature of the physical substance.

Founded more than 150 years ago by German physician and chemist Samuel Hahnemann (1755-1843), the homeopathic principle of healing is based on the premise that a substance that, in high doses, is suffering from a healthy man is able, in small doses, to heal a sick person; a kind of whoever gets or kills does not make you sick - which seems to leave no one indifferent, considering that over time, no other medical practice has provoked so many controversies, criticisms and passions.

Lately, across the world, numerous scientific experiments have confirmed traditional beliefs and intuitions that attribute water to the role of mediator between the energy and the material world, that is, the function of an accumulator, transducer and energy transmitter and information.

But according to Jared Smith, of the Lawrence Berkeley National Laboratory, California, water cannot store information, because this would contradict almost everything scientists know about the ultra-dynamic structure of liquids, in fact, the structure of water evolving faster than any other known liquid. A simple and apparently convincing, but completely false argument argues Martin Chaplin, a professor at London South Bank University. "There is no doubt that hydrogen bonding is happening and breaking rapidly, but that does not mean it cannot have long-term effects." 
For a long time, researchers have found that under certain conditions an atomic configuration acts as a pattern, causing the appearance of a similar pattern to another chemical. In fact, this phenomenon, called epitaxy, has long been involved in making microchip complexes from semiconductor materials and, according to Rustum Roy of Pennsylvania State University, could also explain the effect of water memory.

In a paper published earlier this year in an American magazine, Professor Roy and colleagues have brought back the water's ability to generate epitaxial effects when it's frozen. Experiments to "call" the rain through the socalled "sowing" of clouds with chemicals (iodine colloid, for example) have revealed the ability of ice crystals to take the shape of the molecule from the chemical compounds, suggesting that such a phenomenon would also spend with liquid water. Lionel Milgrom, a chemist at Imperial College London, said he could imagine such a process. "It's difficult to see because water is a fluid, but individual water molecules are connected to the grid via intermolecular hydrogen bonds. It is supposed that this could produce a cloud dynamic effect of trillions of water molecules, all of which respond to each other in a coherent way."

In turn, Swiss chemist Louis Rey used a process called thermo luminescence to analyze the light emitted by highly diluted solutions of compounds in water. Some of the water samples were so diluted that they did not even contain a single atom of the original compound, but even so, their thermo luminescent "imprint" was homogeneous, the compounds being in some way "memorized" by the water.

Recently, at Stanford University, the United States, to study the chemical bonds in the water molecule, a team of researchers used X-rays produced by a sort of particle generator called synchrotron and concluded that liquid water has a structure in totally disagree with what has been thought for over a century. Instead of being a "big" small "pyramid" with the trihedral tetrahedron (tetrahedrons) formed when each water molecule connects with four others, it seems to be an "ocean" made up of "rings and chains", most of the molecules coupling only with two others. The idea of the Stanford scientists has failed to convince the scientific world by far, but this has not prevented a Los Angeles company from launching "the world's first vibrating bottled water a few months ago." Although H2Om is nothing more than what we normally call the cold water, it seems that the "exposure" to the words on the label is able to induce, as the case may be, the state of "love" or " perfect health, "the first two" varieties "of $\mathrm{H} 2 \mathrm{Om}$ launched on the market transmitting each" vibrational "frequency absorbed by water.

William Tiller, a technology specialist at Stanford University, is also pleading in a recent article on the idea that water can really change its properties and structure.
Checking more than 100 studies, he came to the conclusion that water is "a kind of zoo" in which "animals" are molecules of different sizes.

"The world has ignored the possibility that liquid water has multiple structures," says Rustum Roy of Pennsylvania State University, but "there is valid evidence that nanostructures are in it"; as such, in a tube measuring between one and two nanometers, containing between 60 and 80 water molecules, interactions with the container wall could change the structure of the water.

But the surprises that the Earth's most abundant substance is still on are not over yet.

Among the most bizarre statements made about water are those of Masuro Emoto (author of the bestseller Messages from Water, published in Japan in 1999). According to Emoto, water can be affected by the good or bad intentions of the people they "come in contact" with.

The evidence consists of photographs of some ice crystals that Masuro says are "wonderful" when they come from the blessed water of zen monks and "horribly distorted" when water was "exposed" to the influence of unclean intentions. A pilot study by a team of researchers at the Institute of Noetic Sciences in California tried to prove the validity of the Japanese claims and the results (published in October this year) were able to delight those convinced by the properties mystical water.

The comparative analysis of the aesthetic qualities of ice crystals made up of "exposed" water for five minutes to a group of 2,000 Japanese and crystals made of "cold" water revealed that the "blessed" are well superior to the crystals in the second category. For some, the most eloquent example of the water's ability to memorize energy information is the water sources found throughout Europe in the Marian sanctuaries - sites traditionally recognized as sacred, where Virgin Mary's appearances were announced and it is said that there have been many miraculous healings.

About the waters of Lourdes (France), Medjugorje (Croatia), Fatima (Portugal), Montichiari and San Damiano (Italy) are believed to have recorded in them the frequencies of the spiritual energy associated with each of these sanctuaries. Recently, scientific analyzes at the University of Milan have shown that Marine waters possess unique properties that can be transferred to normal water by successive dilutions after small amounts of these waters added to the sink water have changed the $\mathrm{pH}$, conductivity and potential oxidation of the latter.

Water is the universal environment of all biological activities. Almost two-thirds of the human body and about half of each human body, in volume, consist of water.

Water is tremendously frightening when it needs to heat up or cool down. A "defect" that makes the oceans of the world very effective in redressing unexpected climate changes by braking temperature changes. 
Expansion of water as freezing allows ice pieces to float, preventing the oceans from freezing high from bottom to bottom and killing the entire marine life.

Hydrogen bonds between water molecules are extraordinarily flexible, allowing molecules to travel at high speeds inside and outside cells through channels called aquaporin.

Transforming water into vapors requires a lot of heat, which helps protect living dehydration bodies when the weather is very hot and underpins the process called sweat.

Electrostatic changes from water molecules enable the latter to play a key role in creating amino acids (acids that are the foundation of all living organisms).

The water is almost perfectly transparent to visible light but strongly absorbs infrared radiation, making the Earth a place full of light and warmed by 30 degrees Celsius than the surrounding interplanetary space.

Whatever the truth about water memory, recent studies confirm new fundamental functions of this fluid in the process of life. Many of them are based on the so-called hydrogen bonding properties of neighboring water molecules.

The water inside the living cells is highly structured, arranged in various intermolecular forms and the extensive hydrogen bonding networks that structure them give the water properties and behavior very different from "loose" water. Though stronger than most intermolecular bonds, hydrogen bonds are still too weak to allow water molecules to have extraordinary flexibility. This property is vital for living cells, where molecules guide the creation of proteins, ensuring proper functioning.

Until that time, one of the most respected French scientists, Benveniste (deceased in 2004), has become, as a result of his theories, the "black sheep" of the French scientific world. Marginalized, he lost his government subsidies and lab, but, along with a small team of researchers, he continued to investigate the biological effects of agitated and highly diluted solutions. The following results of Benveniste were considered by most biologists and less credible than previous ones. In 1999, he explained, in front of an audience of specialists (including two Nobel Prize laureates), that the effects of biologically active molecules-such as adrenaline, nicotine and caffeine-but also the immunological signatures of viruses and bacteria can be recorded and sent "to work" anywhere in the world via the Internet. According to Benveniste's explanations, all biological reactions occur in water and water molecules completely surround any other molecule placed between them; as such, a single protein molecule, for example, will have a fan club of at least 10,000 water molecules, highly entertaining "admirers," who transmit and amplify the biological signal coming from the original molecules.

It is like a $\mathrm{CD}$, which in itself cannot produce any sound, but it has the means to do it, being engraved on its surface; in order for the sound to be heard, it must pass through an electronic amplifier and, just as Pavarotti or Elton John is on the CD only as "memory", so the water can memorize and amplify the signals of the diluted/dissolved molecules in it. Molecules should not be present in water, they get the "footprint" they leave on the solution they were dissolved in because the agitation activates the "memory". Had he been able to prove that what he says is true, Benveniste would no doubt have won a Nobel. But this did not happen.

In antiquity, the Greeks have found magnetic rocks in the Magnesia (Asia Minor) area now belonging to Turkey. These have been discovered in the form of rocks called magnetite.

Name is under which are known today magnets come from the name of Magnesia. Magnetite is also found today in the mountains of Norway, Sweden, the island of Elba and other areas of the earth's crust.

Bodies that, under certain conditions, attract metals from the iron family are called magnets and their property is called magnetic property. By attaching a piece of iron to a magnet, without touching it, we notice that the iron is actuated by any end of the magnet. Interestingly, it is the fact that of this piece of iron is then caught needles, nails, some coins, etc. In other words, the iron piece acquires magnetic properties as long as it is attracted to a magnet. These experiences demonstrate that there is a magnetic field around the magnets, whose properties and materiality are highlighted by attracting iron objects and by orienting the iron filing near the magnets.

Magnetism is one phenomenon that manifests itself through forces of attraction or rejection between bodies; magnetic forces originate in the movement of electrons or other charged particles. When magnetism is produced by free electric charges, for example in electric current, in plasma or in electrically charged particles, the phenomenon is called electromagnetism. Electrons in orbital motion in the atom produce magnetism; it is more easily observed in permanent magnets, for example in natural minerals such as magnetite (an iron oxide, $\mathrm{Fe}_{3} \mathrm{O}_{4}$ ) or in iron and some of its alloys (including a part of steels) that can be magnetized. Permanent magnets (materials that can be magnetized by an external magnetic field and have the property of staying magnetized and emitting magnetic fields even after removing the external field) have persistent magnetic fields caused by the effect called ferromagnetism. Magnetism also manifests itself as magnetic liquids.

The concept of spin for elementary particles was initially proposed by Ralph Kronig, George Uhlenbeck and Samuel Goudsmit in 1925 as a particle rotation around its own axis. In quantum mechanics and the elementary particle physics, spin is called the intrinsic kinetic momentum of a particle (electron, proton, atom, ...) 
In classical mechanics, the angular impulse of a body is associated with the rotation of the body around its own mass center. In quantum mechanics, spin is particularly important for atomic-sized systems, such as atoms, protons, or electrons. Such particles have certain nonclassical characteristics and for them, the intrinsic angular impulse cannot be associated with rotation but only refers to the presence of the angular impulse.

Stern and Gerlach (1922) sought to measure the magnetic moments of individual atoms by studying the behavior of a beam of atoms in a non-homogeneous magnetic field and trying to verify the theoretical formula: $\mu \mathrm{z}=-\mathrm{m} \mu \mathrm{b}$ (where $\mu \mathrm{z}$ is the projection of the orbital magnetic moment and $\mu b$ is magneton Bohr-Procopius). In their experiment, a beam of silver atoms obtained by means of an oven moves along the direction of the $\mathrm{x}$-axis, encountering a magnetic field oriented along the $\mathrm{z}$-axis, ie perpendicular to the direction of movement of the atoms.

This magnetic field possesses great inhomogeneity in space and consequently on the poles of the atomic magnetic dipole there are forces of different sizes, the result of which imparts to the dipole acceleration either in the sense of the field or in the opposite direction, depending on the orientation of the dipole in relation to the field. If the atomic dipole were in a homogeneous magnetic field, the resulting force that would act on it would be null, because the magnetic force acts on its northern pole with the same intensity as its south pole. It has been shown that atoms in the state possess all the same magnetic moment and its projection on the selected axis can take only two values: $\mu \mathrm{z}= \pm \mu \mathrm{b}$.

By the Einstein-De Haas experiment, a ferromagnetic bar, suspended by a quartz wire, is magnetized by a current passing through the coil, the bar making then torsion oscillations. Experimental measurement of the gyromagnetic factor led to the following results.

The ratio $\gamma=-$ ge $/ 2 \mathrm{~m} 0$ has a negative value, indicating that magnetization of the ferromagnetic bar is due to electron movement;

The value of Lande, $\mathrm{g}$, is not equal to 1 according to Schrodinger's theory but equals two.

To explain the results of the two experiments, Uhlenbeck and Goudsmidt (1925) hypothesized that the electron possesses, in addition to orbital moments, its own kinetic and magnetic moments. These moments have been called electronic spin in connection with trying to link them to the electron rotation movement around its own axis. The kinetic moment of the electron is: $|s|=s \hbar=1 / 2 \hbar$, so the projection is: $\mathrm{sz}=\mathrm{ms}= \pm 1 / 2 \hbar$. After the electron spin was introduced, both the magnetic properties of the substances and the multiple structures of the spectral lines emitted by the atoms were based (Wichmann, 1983; Tomonaga, 1997; Mânzatu, 1969; Halliday and Robert, 1966).

Atoms lack a well-defined outer boundary, so their dimensions are usually described in terms of atomic radii. This is a measure of the distance that the electron cloud extends from the nucleus. This presupposes, however, that the atom would present a spherical form, which is true only for the atoms in the void or in the free space. Atomic rays can be calculated from the distances between two nuclei when two atoms are joined in a chemical bond.

The radius varies depending on the location of an atom in the atomic structure, the type of chemical bond, the number of neighboring atoms (coordinate number) and the quantum mechanical property called spin. In the Periodic Table of Elements, the size of atoms tends to increase when we move down the columns, but decrease when moving on the rows (from left to right). As a result, the smallest atom is helium, with a radius of 32 $\mathrm{pm}$, while one of the largest is cesium, with $225 \mathrm{pm}$.

In some papers (Halliday and Robert, 1966; Kramer, 2011; Krane, 1987; Moses et al., 2009; Petrescu and Calautit, 2016a-b; Petrescu and Petrescu, 2014, 2019; Petrescu et al., 2016; Petrescu, 2012a-b, 2014, 2019; Shultis and Faw, 2002; Figgis and Lewis, 1960) it has been shown that these dimensions of atoms and subatomic particles are measured at approximately average values and for low displacement rates of the particles. Dynamically, the dimensions of these particles vary greatly with the variation in their displacement speed.

The same thing happens with the energies of these permanently moving particles. For this reason, in the present paper, we propose to briefly determine the rotational kinetic energy of such a particle, depending on its linear displacement velocity and obviously its resting mass.

In all these processes it is necessary to know the basic parameters of the physical particles (elementary or not), including their dimensions and the speeds with which they move and rotate around their own axis (Halliday and Robert, 1966; Kramer, 2011; Krane, 1987; Moses et al., 2009; Petrescu and Calautit, 2016a-b; Petrescu and Petrescu, 2014, 2019; Petrescu et al., 2016; Petrescu, 2012a-b, 2014, 2019; Shultis and Faw, 2002).

\section{Materials and Methods}

Ferromagnetism (including ferromagnetism) is the most common and most powerful type of magnetism responsible for what is commonly called the magnetic phenomenon. Not all substances are ferromagnetic, but only certain metals such as iron, nickel, cobalt and most of their alloys, which form permanent magnets by magnetizing them or are attracted by magnets.

Other substances respond very poorly to the magnetic field under the action of two other forms of magnetism, namely paramagnetism and diamagnetism, but the forces are so weak that they can only be detected by sensible laboratory instruments.

Paramagnetism is a form of magnetism, manifested only in the presence of an external magnetic field. 
In the absence of the outer magnetic field, the atoms of the paramagnetic materials have their own magnetic moment different from zero. Paramagnetic substances introduced into a magnetic field are poorly attracted. The force of attraction is proportional to the intensity of the magnetic field. Depending on the thermal agitation, the atomic moments of these substances can be oriented in a magnetic field.

Diamagnetism is the property of an object, which is manifested by the appearance of a magnetic field in opposition to a magnetic field applied externally. More specifically, an external magnetic field changes the rotational speed of electrons around the atomic nucleus, thus changing the magnetic moment of the magnetic dipole in a direction opposite to the direction of the outer magnetic field. Diamagnetic materials are materials with magnetic permeability less than $\mu 0$ (a relative permeability less than 1).

Therefore, diamagnetism is a form of magnetism that manifests in the presence of an external magnetic field. It is generally quite weak on most materials, unlike superconductors, which have a strong effect.

A ferrofluid or magnetic fluid is a fluid composed of ferromagnetic, ferromagnetic, or paramagnetic colloidal particles suspended in a carrier liquid. Ferrofluids react to external magnetic fields. The dispersed particles of ferrofluid consist of magnetite, hematite, or another iron compound. Magnetic fluids have surfactants to prevent agglomeration.

Magnetorheological fluids are fluids that can modify their viscosity by the action of an external magnetic field. They have great practical importance.

An ionic, magnetic fluid is a magnetic fluid constituted by an ionic liquid that reacts to the action of an external magnetic field. An example of such a liquid is the one discovered by two Japanese in 2004 based on pyrrole and ferric chloride derivatives.

Fusible alloys are alloys with melting points at ambient temperatures. An example would be those based on gallium.

The thermomagnetic engine is a motor that works by heating a ferromagnetic material above the Curie point when demagnetizing. Experiments have produced only highly inefficient prototypes of work. The principle of the thermomagnetic engine has been studied as a possible actuator in so-called "intelligent" materials.

Magnetochemistry is a branch of physical chemistry with the object of studying the magnetic properties of chemical compounds. It was initiated by Paul Langevin (Figgis and Lewis, 1960).

The first permanent magnetic fluid, recently and accidentally obtained in the lab, makes us very much nervous because researchers today do not yet understand the mechanism of this astounding discovery.

A team of researchers at the University of Massachusetts Amherst has accidentally obtained the first magnetized permanent liquid in the world, the droplets of this liquid moving in unison and able to associate in different forms while being manipulated exterior by means of a magnet, according to a new study published in the latest issue of the journal Science, reports Agerpres.

We usually imagine magnets as being in a solid-state of aggregation, "says Thomas Russell, professor of polymers and engineering at the University of Massachusetts Amherst. But we now know that "we can get liquid magnets that can take different forms - and we can decide the forms they take," he added, specifying that droplets in this magnetic fluid can form spheres, cylinders, or the shape of a flat disc of the pancake dough. "We can make them look like a sea hedge if we want," he said.

Russell and his team obtained this liquid magnet by chance while experimenting with a 3D printer. They have tried to print liquids in order to obtain new solid materials, but having the properties of fluids, for various applications in the field of energy. One day, the postdoctoral student and coordinator of this study, Xubo Liu, noticed that droplets of the resulting 3D material, made up of magnetized iron oxide particles, rode unison on magnetic support. Subsequently, his team noticed that the entire construct, not just those particles, had become magnetic. Using a specially customized 3D printer for liquids, the team managed to generate millimeters drops of water, oil and iron oxides. These drops retain their shape because of some of the iron oxide particles in the composition form bonds with the surfactants (also called tensides substances that reduce the surface tension of the liquid). Surfactants form a film around the water and some iron oxide particles enter the composition of the film, while the rest remain inside, as Russell explains. Then the team placed these droplets near a magnetic coil to magnetize them. After removing the magnetic coil, droplets have shown unprecedented behavior to liquid substances so far - they have retained their magnetic properties. Magnetic fluids were known to physicists.

They are called ferrofluids and are fluids consisting of ferromagnetic, ferromagnetic or paramagnetic colloidal particles suspended in a carrier liquid. Characteristic of ferrofluids is that they remain magnetized only in the presence of a magnetic field. When the droplets were close to the magnetic field, the iron oxide particles aligned unison, indicating the same direction.

Once the magnetic field has been stopped, the iron oxide particles have joined the surfactant in the film in tight formation, without being able to move and thus keep their alignment. The iron oxide particles that have remained floating inside the liquid have also taken this alignment. Scientists still do not understand the mechanism by which these particles remain magnetized. Once they have this explanation, the applications of this discovery can be multiple, both in the field of energy, robotics and space programs.

The rotational kinetic energy of a subatomic particle can be determined by the relationship (1), where $\mathrm{c}$ is the light velocity, $\mathrm{h}$ represents the Plank constant, $\mathrm{v}$ is the 
velocity of the particle in its linear movement and $\mathrm{m}$ its mass in linear movement (Krane, 1987; Petrescu, 2019; Wichmann 1983):

$$
\omega=\frac{2 \pi \cdot c}{h} \cdot m \cdot v
$$

You can immediately get the square of the angular velocity of the particle (2):

$$
\omega^{2}=\frac{4 \pi^{2} \cdot c^{2}}{h^{2}} \cdot m^{2} \cdot v^{2}
$$

The mass at the rotation movement of the particle (considered by the spherical shape) is determined by the relationship (3):

$$
J=\frac{2}{5} \cdot m \cdot R^{2}
$$

The kinetic energy to the particle rotation is obtained with relation (4):

$$
\begin{aligned}
& E_{c \omega}=\frac{1}{2} \cdot J \cdot \omega^{2}= \\
& \frac{1}{2} \cdot \frac{2}{5} \cdot m \cdot R^{2} \cdot \frac{4 \pi^{2} \cdot c^{2} \cdot m^{2} \cdot v^{2}}{h^{2}}= \\
& \frac{4}{5} \frac{\pi^{2} \cdot c^{2}}{h^{2}} \cdot m^{3} \cdot v^{2} R^{2}
\end{aligned}
$$

The particle radius to the square can be calculated with the original relation (5):

$$
R^{2}=\frac{5}{4} \cdot \frac{h^{2}}{\pi^{2} \cdot c^{2}} \cdot \frac{c^{2}-c \sqrt{c^{2}-v^{2}}-\frac{1}{2} v^{2}}{m^{2} \cdot v^{2}}
$$

With the expression of the radius of the relationship (5), the kinetic energy at the rotation of particle from the relationship (4) gets the form (6), where $\beta$ represents the ratio between $\mathrm{v}$ and $\mathrm{c}$ (relation 7 ):

$$
\begin{aligned}
& E_{c \omega}=m \cdot\left(c^{2}-c \sqrt{c^{2}-v^{2}}-\frac{1}{2} v^{2}\right)= \\
& m \cdot c^{2} \cdot \frac{c^{2}-c \sqrt{c^{2}-v^{2}}-\frac{1}{2} v^{2}}{c^{2}}= \\
& m \cdot c^{2}\left(1-\sqrt{1-\beta^{2}}-\frac{1}{2} \beta^{2}\right) \\
& \beta=\frac{v}{c}
\end{aligned}
$$

It can be seen from the relationship (6) that the kinetic energy of rotation of the particle represents a percentage of its total energy. It can also be expressed as another percentage of its resting energy (relationship 8):

$$
E_{c \omega}=m_{0} \cdot c^{2} \cdot \frac{1-\sqrt{1-\beta^{2}}-\frac{1}{2} \beta^{2}}{\sqrt{1-\beta^{2}}}
$$

Finally, one holds for the kinetic energy at rotation of the particle only the system relations (9):

$$
\left\{\begin{array}{l}
E_{c \omega}=m \cdot c^{2}\left(1-\sqrt{1-\beta^{2}}-\frac{1}{2} \beta^{2}\right) \\
E_{c \omega}=m_{0} \cdot c^{2} \cdot \frac{1-\sqrt{1-\beta^{2}}-\frac{1}{2} \beta^{2}}{\sqrt{1-\beta^{2}}}
\end{array}\right.
$$

\section{Results and Discussion}

All materials exhibit diamagnetic behavior under the action of an external magnetic field. In fact, diamagnetism is a very general phenomenon, because all the pair electrons, including base electrons (electrons not on the valence layer), will always make a slight contribution to the diamagnetic response of the material. However, for materials having another form of magnetism (such as ferromagnetism or paramagnetism), diamagnetism is very weak. Substances which are particularly diamagnetic in nature are called diamagnetic materials or diamagnetics. Diamagnetic materials are those that "non-physicists" generally consider being non-magnetic and include water, wood, most organic compounds such as petroleum and some plastics and many metals including copper, especially heavy metals many basic electrons, such as mercury, gold and bismuth.

The diamagnetic materials have a relative magnetic permeability less than 1 , so the magnetic susceptibility is less than 0 and are therefore rejected by the outer magnetic fields. However, because diamagnetism is a weak property, its effects are not observable in everyday life.

The magnetic moment is a physical magnitude that determines the magnitude of the moment of force that a magnet is subjected to in the external magnetic field. It is a vector oriented from the south pole to the north pole of the magnet. Power loop, beam magnet, an electron in motion around the nucleus, molecule, planet have a magnetic moment

The dipole or electrical dipole moment is a vectorial physical magnitude that characterizes a spatial polarized distribution of electrical charges. The dipole moment is a measure of separating the positive and negative electric charges (charges) into an electrical charge system and thus a measure of system polarization. It is important for the characterization of chemical bonds. An electrical dipole is a system consisting of two electrical charges having the same absolute value but a countersign (contrary to polarity). The unit of measurement in the International System is Coulomb-Meter (CM) and in the CGS system is Debye (D). 
In atomic physics, the gyromagnetic ratio of a particle is defined as the ratio between the magnetic moment and the kinetic moment of the particle.

The new discoveries in magnetism and recently in the magnetism of liquids bring great hope to future applications related to magnetic or electromagnetic fields.

There is more talk about the possibility of using water as an energy support due to the current possibility of separating water in its hydrogen and oxygen components with a very low energy consumption by modern methods, using nanotechnologies, passing water under pressure through nano filters to be broken atomic in its components with ease, using precious metal catalysts and irradiation with ultraviolet radiation at the same time.

Such applications could produce in the future energy to infinitely in micro home-owned, factories, enterprises, institutions, or mounted on different types of vehicles.

Another major possibility of getting infinite power on small power plants or on different types of vehicles is the use of magnetic motors, already tested for about 20 years.

The major problem that arises with such magnetic motors is the fairly rapid demagnetization of the materials used and tested to date. An international army of scientific researchers is constantly working on the situation, but clear remedies could not yet be obtained even though more modern and intelligent magnetic materials have already been subjected to various tests.

If we have recently learned that liquids can be magnetized, we will now seriously think about starting new magnet and magnetic motor tests to which other magnetic materials also contribute to better magnetization. The future sounds better now and we will soon find out how magnetic (magnetized) fluids can be used in future businesses.

A team of scientists from the University of Massachusetts Amherst accidentally obtained the first permanently magnetized fluid in the world, the droplets of this fluid movement in unison and may associate in different forms while being externally manipulated by a magnet, according to a new study published in a recent issue of the journal Science.

"We usually imagine that magnets are in a solid-state of aggregation," said Thomas Russell, Professor of Polymer Science and Engineering at the University of Massachusetts Amherst. But now we know that "we can get liquid magnets that can have different shapes - and we can decide the shapes they take," he added, specifying that droplets in this magnetic fluid can form spheres, cylinders, or the shape of a flat pancake. "We can make them look like a hedge if we want," he said.

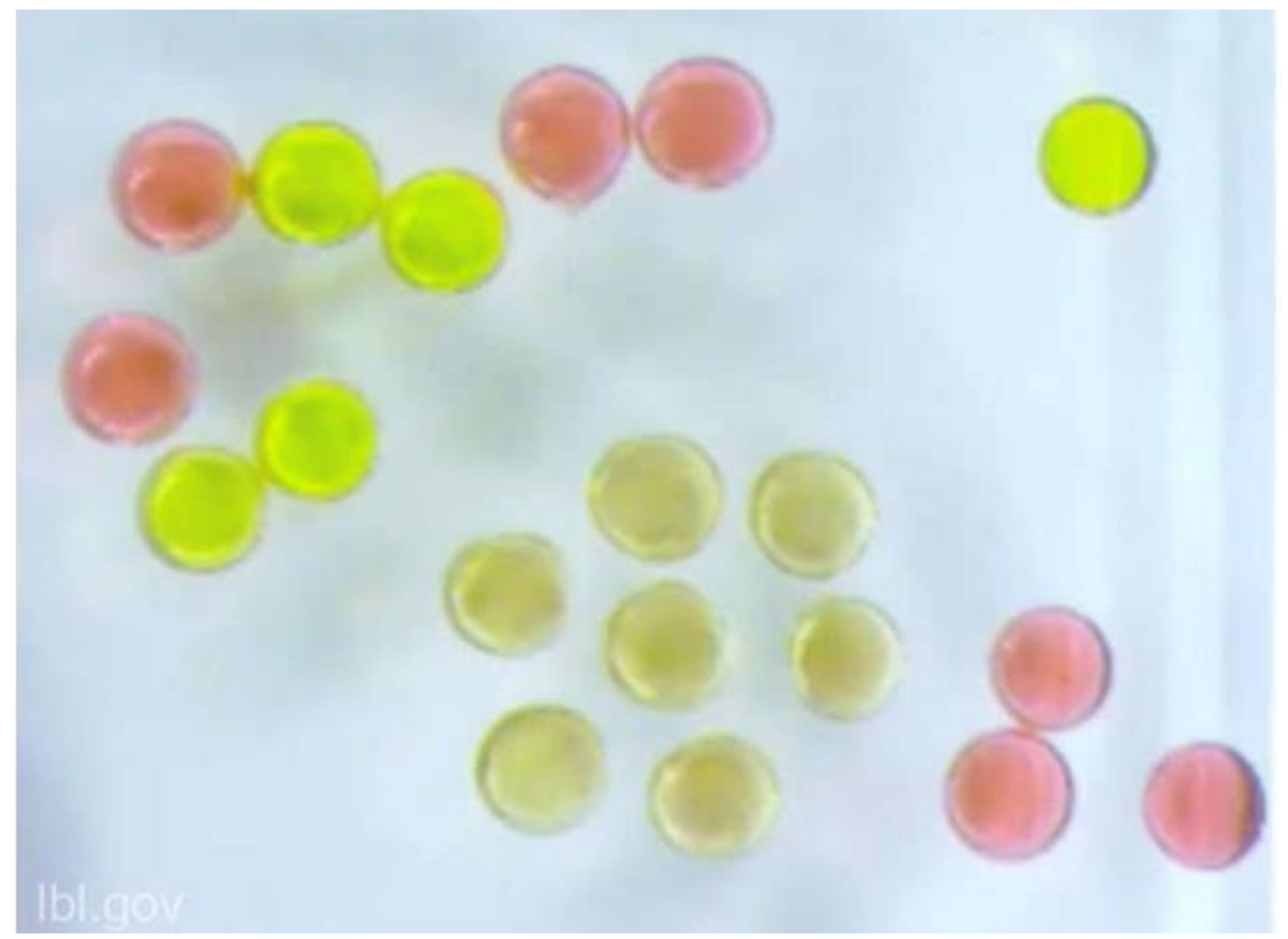




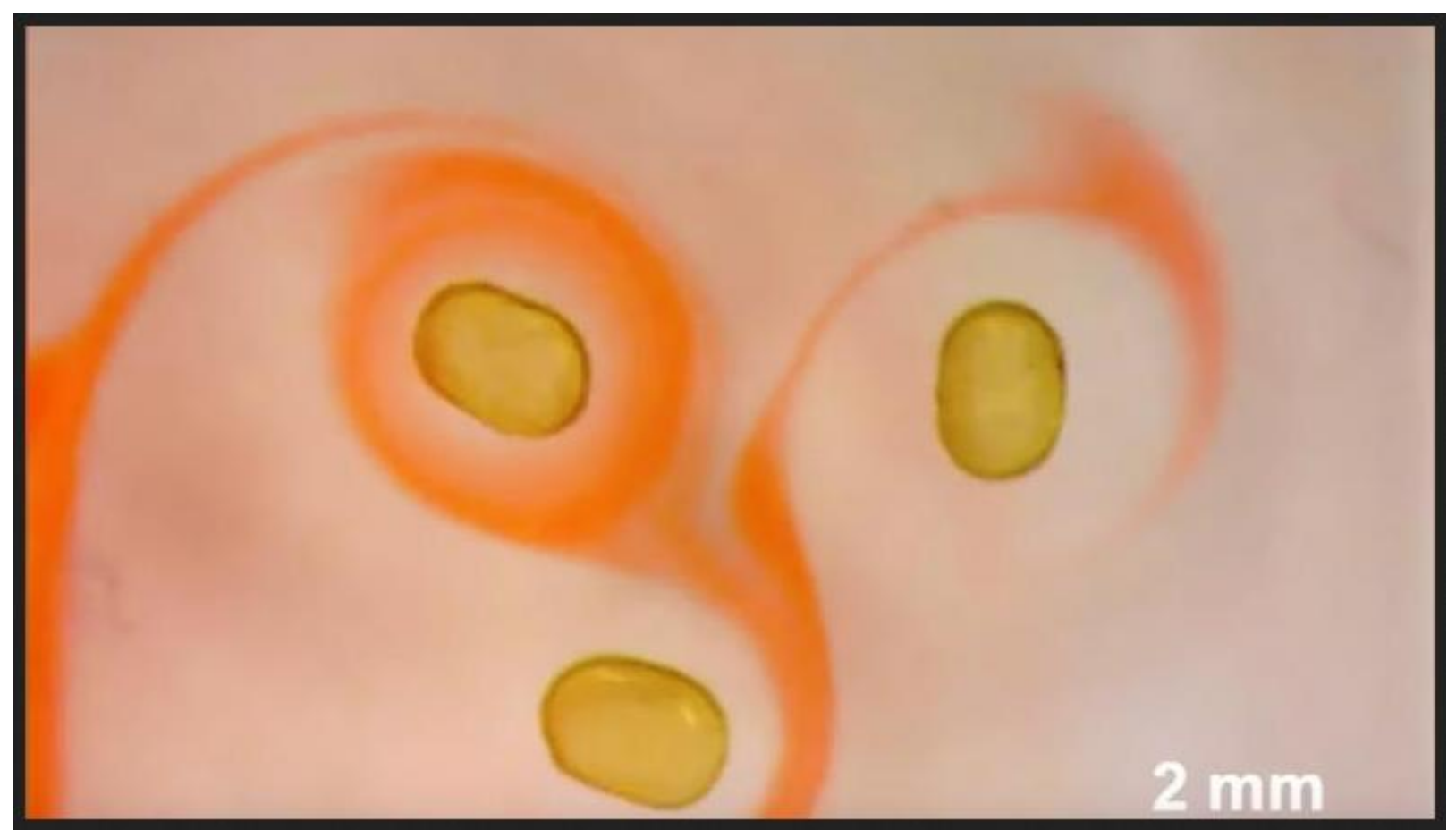

Fig. 1: The first permanently magnetized liquid recently discovered (Berkeley Lab, 18 July 2019)

Russell and his team obtained this liquid magnet by chance while experimenting with a $3 \mathrm{D}$ printer. They have tried to print liquids to get new solids but have fluid properties for different energy applications.

One day, the postdoctoral student and coordinator of this study, Xubo Liu, noticed that droplets resulting from 3D material, made up of magnesium iron particles, led to magnetic support. Subsequently, his team noticed that the entire construct, not just those particles, had become magnetic.

Using a 3D Fluid Printer, the team managed to generate droplets of water, oil and iron oxides in millimeters. These drops retain their shape due to the fact that some of the iron oxide particles in the composition bind to surfactants (also called tensides-substances that reduce the surface tension of the liquid). Surfactants form a film around the water and some iron oxide particles enter the composition of the film, while the rest stays inside, explains Russell.

Then the team put those drops near a magnetic coil to magnetize them. After removing the magnetic coil, the drops exhibited unprecedented fluid behaviors so far-they retained their magnetic properties. Magnetic fluids were known to physicists.

They are called ferrofluids and are fluids consisting of ferromagnetic, ferromagnetic or paramagnetic colloidal particles suspended in a carrier liquid. The characteristic of ferrofluids is that they remain magnetized only in the presence of a magnetic field.
When the droplets are close to the magnetic field, the iron oxide particles align in one direction, indicating the same direction.

Once the magnetic field has been stopped, the iron oxide particles have been bonded to the film-bound surfactant in tight form without being able to move and thus maintain alignment. The iron oxide particles that remained floating inside the liquid also took this alignment (Fig. 1), (Berkeley, 2019).

\section{Conclusion}

A The diamagnetic materials have a relative magnetic permeability less than 1 , so the magnetic susceptibility is less than 0 and are therefore rejected by the outer magnetic fields. However, because diamagnetism is a weak property, its effects are not observable in everyday life.

The magnetic moment is a physical magnitude that determines the magnitude of the moment of force that a magnet is subjected to in the external magnetic field. It is a vector oriented from the south pole to the north pole of the magnet. Power loop, beam magnet, an electron in motion around the nucleus, molecule, planet have a magnetic moment

The dipole or electrical dipole moment is a vectorial physical magnitude that characterizes a spatial polarized distribution of electrical charges. The dipole moment is a measure of separating the positive and negative electric charges (charges) into an electrical charge system and 
thus a measure of system polarization. It is important for the characterization of chemical bonds. An electrical dipole is a system consisting of two electrical charges having the same absolute value but a countersign (contrary to polarity). The unit of measurement in the International System is Coulomb-Meter (CM) and in the CGS system is Debye (D).

In atomic physics, the gyro magnetic ratio of a particle is defined as the ratio between the magnetic moment and the kinetic moment of the particle.

The new discoveries in magnetism and recently in the magnetism of liquids bring great hope to future applications related to magnetic or electromagnetic fields.

If we have recently learned that liquids can be magnetized, we will now seriously think about starting new magnet and magnetic motor tests to which other magnetic materials also contribute to better magnetization. The future sounds better now and we will soon find out how magnetic (magnetized) fluids can be used in future businesses.

\section{Acknowledgement}

This text was acknowledged and appreciated by Dr. Veturia CHIROIU Honorific member of Technical Sciences Academy of Romania (ASTR) Ph.D. supervisor in Mechanical Engineering.

\section{Funding Information}

Research contract: Contract number 36-5-4D/1986 from 24IV1985, beneficiary CNST RO (Romanian National Center for Science and Technology) Improving dynamic mechanisms internal combustion engines.

Copyrights:

1. New Aircraft (New Ionic or Beam Engines): no. 548 of 22-04-2010 [cgiywDssin], Aerospace Engineering

2. Some Few Specifications About the Doppler Effect to the Electromagnetic Waves: 636 of 28-05-2010 [iEtcaouxxA], physics

3. Presenting an Atomic Model and Some Possible Applications in LASER Field: nr. 639 of 29-052010 [yncngrotfo], physics

4. Some Applications in LASER Field: no. 718 of 0907-2010 [xeujouincC], physics

5. The Energies of Today and Tomorrow: nr. 819 of 30-09-2010 [kbHquxwykr], energy engineering

6. Obtaining Energy by the Annihilation of the Matter with Antimatter - The Battle for Energy: nr. 1068 of 13.03.2011 [GfEqpGDzeh], Energy Engineering

\section{Author's Contributions}

All the authors contributed equally to prepare, develop and carry out this manuscript.

\section{Ethics}

This article is original and contains unpublished material. Authors declare that are not ethical issues and no conflict of interest that may arise after the publication of this manuscript.

\section{References}

Berkeley, L., 2019. Scientists Print Liquid Magnetic Droplets.

https://newscenter.lbl.gov/2019/07/18/new-laws-ofattraction-scientists-print-magnetic-liquid-droplets/

Figgis, B.N. and J. Lewis, 1960. The magnetochemistry of complex compounds. lewis. J. Wilkins. R.G. Modern Coordination Chemistry. New York: Wiley.

Halliday, D. and R. Robert, 1966. Physics, Part II. 1st (Edn.), John Wiley and Sons, Inc., New York.

Kramer, D., 2011. DOE looks again at inertial fusion as a potential clean-energy source. Phys. Today, 64: 26 DOI: $10.1063 / 1.3563814$

Krane, K.S., 1987. Introductory nuclear physics. Proceedingds of the 3rd (Edn.), Wiley and Sons, New York, ISBN-10: 047180553X, pp: 864.

Mânzatu, I., 1969. Polarization and orientation in nuclear physics: Quantum basics of spinal polarization. Academia RSR Publishing House.

Moses, EI., R.N. Boyd, B.A. Remington, C.J. Keane and R. Al-Ayat, 2009. The national ignition facility: Ushering in a new age for high energy density science. Phys. Plasmas, 16: 041006-041006. DOI: $10.1063 / 1.3116505$.

Petrescu, F.I.T. and J.K. Calautit, 2016a. About nano fusion and dynamic fusion. Am. J. Applied Sci., 13: 261-266. DOI: 10.3844/ajassp.2016.261.266

Petrescu, F.I.T. and J.K. Calautit, 2016b. About the light dimensions. Am. J. Applied Sci., 13: 321-325. DOI: 10.3844/ajassp.2016.321.325

Petrescu, F.I.T. And R.V. Petrescu, 2014. Nuclear Green Energy. I.J.A.P., 10: 3-14.

Petrescu, F.I.T. And R.V.V. Petrescu, 2019. Nuclear hydrogen structure and dimensions. Int. J. Hydrogen Energy, 44: 10833-10837.

DOI: $10.1016 /$ j.ijhydene.2019.02.140

Petrescu, F.I.T. A. Apicella, R.V. Petrescu, S. Kozaitis and R. Bucinell et al., 2016. Environmental protection through nuclear energy. Am. J. Applied Sci., 13: 941-946.

DOI: 10.3844/ajassp.2016.941.946

Petrescu, F.I.T., 2014. Nuclear fusion, Infinite Energy, 20: 44-47.

Petrescu, F.I.T., 2012a. Cold nuclear fusion. Plasma Physics Fusion Technol.

Petrescu, F.I.T., 2012b. Cold nuclear fusion. Create Space publisher, USA. 
Petrescu, F.I.T., 2019. About the nuclear particles' structure and dimensions. Comp. Part. Mech., 6: 191-194. DOI: 10.1007/s40571-018-0206-7

Shultis, J.K. and R.E. Faw, 2002. Fundamentals of nuclear science and Engineering. CRC Press.

Tomonaga, Sin-Itiro, 1997. The Story of Spin. University of Chicago Press.

Uhlenbeck, G. \& Goudsmit, S. Naturwissenschaften (1925) 13: 953. https://doi.org/10.1007/BF01558878

Wichmann, E.H., 1983. The berkeley physics quantum physics course.

\section{Source of Figure:}

https://www.agerpres.ro/data/stiri/lichid_magnetic.jpg

\section{NOMENCLATURE}

$\mathrm{h}=>$ the Planck constant: $\mathrm{h}=6.626 \mathrm{E}-34$ [Js]

$\mathrm{q}=>$ electrical elementary load: $\mathrm{q}_{\mathrm{e}}=-1.6021 \mathrm{E}-$ $19[\mathrm{C}] \mathrm{q}_{\mathrm{p}}=+1.6021 \mathrm{E}-19[\mathrm{C}]$

$\mathrm{c}=2.997925$ $\mathrm{E}+08[\mathrm{~m} / \mathrm{s}]$

$\mathrm{m}_{0}[\mathrm{~kg}]=>$ the rest mass of one particle

$\mathrm{m}_{\text {0electron }}=9.11 \mathrm{E}-31[\mathrm{~kg}]$

$\mathrm{m}_{\text {oproton }}=1.672621898(21) \mathrm{E}-27[\mathrm{~kg}]$

$\mathrm{m}_{\text {0neutron }}=1.674927471(21) \mathrm{E}-27[\mathrm{~kg}]$

$\mathrm{m}_{\text {odeuteron }}=3.34449 \mathrm{E}-27[\mathrm{~kg}]$

$\mathrm{m}_{\text {0triton }}=5.00827 \mathrm{E}-27[\mathrm{~kg}]$ 